Tamara Valčić Bulić ${ }^{*}$

Faculté de Philosophie et Lettres

Université de Novi Sad
UDK : 811.133.1-31.09 Devi A.

DOI: $10.19090 /$ gff.2021.3.293-307

Originalni naučni rad

\title{
DES EFFETS DE LA (NON)FIABILITÉ DU NARRATEUR DU « NOEUD DE VIPÈRES » MAURICIEN
}

Le roman Le Sari vert (2009) d'Ananda Devi, écrivaine franco-mauricienne, relate une histoire de haine et de violence familiales entre un homme à l'agonie d'un côté, et de l'autre, son épouse, décédée depuis longtemps et réapparaissant comme un fantôme, le hantant, puis leur fille et leur petite-fille, celles-ci bien vivantes et assoiffées de vengeance. Devi écrit son roman sous la forme du monologue intérieur d'un seul narrateur, qui, cependant note aussi les paroles et les réactions des deux femmes qui l'assistent. Il s'agit dans cet article de montrer comment est obtenu l'effet à la fois de la non fiabilité du narrateur et de sa monstruosité. Les notions théoriques d'auteur implicite et de narrateur (non) fiable/(in)digne de confiance sont empruntées à Wayne Booth, puis nuancées par quelques autres notions narratologiques plus récentes (distinction d'A. Nünning (1999) entre «non fiable» et «indigne de confiance»; J. Phelan (2005) introduit, quant à lui, trois critères ou axes de la (non)fiabilité : narratif, interprétatif et axiologique). À l'aide de ces notions, on conclut à une certaine fiabilité du narrateur dans le domaine des faits, à son interprétation fréquemment erronée de la psychologie et des motivations des autres personnages et enfin à son indignité sur le plan éthique. Le discours narratorial est donc miné par des contradictions discrètement introduites qui sont autant de signaux de la part de l'auteur implicite, le narrateur lui-même est déjoué et sanctionné à la fin du roman, apparaissant dans toute sa turpitude.

Mots clés : Le Sari vert, Ananda Devi, auteur implicite, narrateur (non) fiable, narrateur (in)digne de confiance, axe narratif, axe interprétatif, axe axiologique.

Le Sari vert (2009) d'Ananda Devi est un roman qui relate une histoire familiale de haine et de violence. Le roman se présente sous la forme d'un monologue intérieur d'un homme qui, se trouvant à l'article de la mort, est pris en charge par sa fille et sa petite-fille. Celles-ci, bien qu'elles semblent être là pour l'accompagner dans ces moments, s'acharnent à lui soutirer l'un de ses

*tamara.valcic.bulic@ff.uns.ac.rs 
plus grands secrets, celui qui concerne la mort de sa femme, mère et grandmère des deux femmes. C'est ainsi que l'accompagnement se transforme en un règlement de comptes entre elles et lui, dans ce "nœud de vipères " $^{1}$ que représente cette famille. L'intérêt du roman, en dehors du thème abordé, réside notamment dans le choix du narrateur, celui-là même qui est à l'origine des souffrances de sa famille, ou, autrement dit, celui dont les normes et les valeurs ne pourraient coïncider ni avec celles de «l'auteur implicite» (W. Booth, 1961) ni d'un lecteur contemporain moyen. C'est pourquoi, après une brève présentation du contexte mauricien et de l'œuvre d'Ananda Devi, nous allons tenter de dévoiler de quelle manière, à l'aide de quels moyens, ce narrateur masculin, bourreau de sa famille, dénude sa propre personnalité et les souffrances infligées à sa femme et à sa fille, autrement dit comment l'auteur implicite parvient, sans recourir au pathétique, ni à une prise de position ouverte contre cet homme, à nous convaincre de la monstruosité de ce personnage-narrateur. Pour parvenir à élucider cette question, nous allons d'abord expliquer brièvement les notions théoriques de «narrateur non fiable» ou «indigne de confiance», et d' " auteur implicite», notions introduites par W. Booth, puis nuancées ou contestées par d'autres théoriciens.

Ananda Devi est une écrivaine née à l'île Maurice en 1957. Cette île, découverte par les Portugais dans les premières décennies du XVI ${ }^{\text {e }}$ siècle, a d'abord fait l'objet de la colonisation hollandaise entre 1598 et 1710 . Les

${ }^{1}$ Le rapprochement que nous faisons ici avec Le Nœud de vipères (1932) de François Mauriac tient tout d'abord au thème abordé : c'est Louis, un brillant avocat et père de famille qui, sous la forme d'une lettre à sa femme, puis de journal, fait une confession sur sa haine, le sentiment dominant développé par le vieillard envers sa famille. Cette haine est complexe : elle est sociale, car il est d'une origine plus modeste que sa femme ; elle est née aussi d'une déception profonde liée à l'unique aventure pré-conjugale de sa femme et au sentiment de ne pas être aimé d'elle ; sa haine est dirigée contre ses enfants même, née d'un sentiment de jalousie à leur égard et s'étend à d'autres personnes de sa famille et de son entourage. Avec la plus grande lucidité, Louis livre son histoire, dévoile tout, même ses pires faits et gestes tout en jugeant très sévèrement et méprisant les autres. Bien que Le Noeud de vipères, contrairement au Sari vert, épargne au lecteur toute violence physique et que pour Louis la conversion soit encore accessible, certains parallèles avec Le Sari vert sont possibles non seulement par rapport au thème du récit mais aussi par rapport à la distance existant entre le narrateur et les normes personnelles du lecteur, ce qui sera développé dans la suite de cet article. 
Hollandais ${ }^{2}$ l'ayant abandonnée en raison des intempéries et des maladies, l'île est entre 1715 et 1810 occupée par les Français, pour enfin passer entre les mains des Britanniques jusqu'en 1968, date à laquelle elle acquiert son indépendance. Cette histoire tumultueuse donne lieu à un brassage de populations sur une île originellement inhabitée, brassage entre les colonisateurs blancs, la population africaine d'esclaves noirs, celle des Indiens (des «coolies ») et des autres peuples venus depuis l'abolition de l'esclavage (1835) remplacer les esclaves noirs dans les plantations de la canne à sucre. Ananda Devi appartient à une famille d'immigrés indiens et a vécu à l'île Maurice jusqu'à l'âge de ses dix-huit ans, dans un environnement multilingue dans lequel elle a appris le créole et le télugu, ensemble avec le français et l'anglais. Devi a fait ses études d'anthropologie à Londres, a séjourné au CongoBrazzaville pendant plusieurs années pour enfin, en 1989, se fixer en France avec sa famille, à Ferney-Voltaire, près de la frontière suisse. Elle-même déclare avoir conscience de la mixité de ses origines et de son identité hybride.

C'est pourquoi il n'est pas étonnant ${ }^{3}$ qu'Ananda Devi ait choisi le français comme sa langue d'écriture : c'est donc en français que depuis sa prime jeunesse elle écrit de la poésie et des nouvelles : Solstices (1977), son premier recueil de nouvelles, est publié alors qu'elle n'a même pas vingt ans. Puis, une dizaine d'années plus tard, elle se lance dans l'écriture romanesque. Depuis, Devi a publié une douzaine de romans dont certains ont reçu des prix prestigieux 4 . Pendant toutes ces années, Devi continue à publier de la poésie et des nouvelles, puis, aussi, un récit autobiographique, des livres pour enfants,

\footnotetext{
2 Comme le dit Ananda Devi dans un entretien avec Alessandro Corio, les Hollandais « ont détruit le dodo et les forêts d'ébène » et pour eux Maurice était "seulement une escale commerciale » (Corio, $2005:$ 146).

3 D'après le témoignage de Devi, à Maurice on gère « le plurilinguisme en adoptant une langue principale et en ne déviant pas de cette volonté » (Corio, 2005 : 147) et pour elle, la langue d'écriture est décidément le français.

${ }^{4}$ Comme Moi, l'interdite (2000, Prix Radio France du livre de l'océan Indien), Ève de ses décombres (2006, Prix des cinq continents de la francophonie, Prix RFO du livre), Le Sari vert (2009, Prix Louis-Guilloux de 2010, Prix Continental, 2010, Prix Armorice, 2011), Manger l'autre (2018, Prix Ouest France Étonnants Voyageurs). La liste des romans et des prix n'est pas exhaustive. Certains des livres de Devi ont également été sélectionnés pour les prix Renaudot, Fémina, etc.
} 
mais elle travaille aussi comme traductrice. Il est aussi à noter qu'elle est signataire du manifeste Pour une littérature-monde en français (2007) ${ }^{5}$.

Il n'est guère étonnant non plus que l'action des romans et des nouvelles de Devi se situe principalement à Maurice, île à laquelle elle reste profondément attachée en dépit de son existence de nomade. Dans ses œuvres, bien loin de tout exotisme ${ }^{6}$, elle met souvent en scène des personnages confrontés à une hiérarchie sociale essentiellement patriarcale, à l'exclusion et la souffrance des parias de la société, et ne cesse de dénoncer l'exploitation de l'homme par l'homme, la violence sous toutes ses formes et celle à l'égard des femmes tout particulièrement.

Ainsi, dans Le Sari vert le lecteur découvre une des faces de cette violence: le docteur Bissam Sobnath, pour ses patients «Dokter-Dieu»7, appartient à la communauté indienne de l'île Maurice. Même si le lecteur assiste à ses dernières heures, la véritable histoire se situe dans le passé que le docteur est en train de revivre et de rêver dans son demi-délire, car, comme il le constate lui-même, «le ricanement des souvenirs que l'on croyait enterrés est effroyable » (Devi, 2009: 95). Il s'est marié avec une très jeune femme, jamais nommée dans le roman ${ }^{8}$, appartenant à la haute société contrairement au docteur, qui est, lui, orphelin de père ayant vécu avec sa mère dans une «inconsolable pauvreté» (Devi, 2009: 97). De cette union, qui s'est vite dégradée et s'est transformée en un mariage-torture et séquestration ${ }^{9}$, sont

${ }^{5}$ Cf. à propos du manifeste, Valčić Bulić, T. (2017). De la négritude à la littératuremonde : des mouvements identitaires à une communauté (littéraire) dénationalisée, Godišnjak Filozofskog fakulteta u Novom Sadu, XLI-3, 217-233.

${ }^{6}$ D'après Devi elle-même, certaines des œuvres de ses débuts ont été refusées par les éditeurs en raison de l'absence d'exotisme et de «couleur locale» (http://www.latortueverte.com/Entretien\%20avec\%20Ananda\%20Devi.pdf).

${ }^{7}$ L'italique est de nous.

8 Le mari ne désigne sa femme que par des qualificatifs comme "innommée», «l'autre », «la mère », « cette femme », "sa majesté ma femme ». Cependant, elle est aussi métonymiquement désignée dans le titre par le sari vert, à la fois signe de sa féminité et de sa beauté (le sari vert étant un de ses vêtements préférés) et de son enfermement dans son rôle de femme tout comme le symbole de sa fin tragique. À propos du sari, cf. Wiśniewska, (2011: 316).

${ }^{9}$ La jeune femme est traitée de plus en plus durement, puis exposée à des ordres et des interdictions, des gifles, des coups de poing, à du riz gluant et brûlant renversé sur la tête. La haine s'installe des deux côtés: "une extraordinaire haine blanche dont l'odeur même était masculine et fécale » (Devi, 2009 : 30-31). 
nés deux enfants : une fille, Kitty - de son vrai nom Kaveri Bhavani -, puis un garçon, celui-ci ayant dépéri très vite après sa naissance. La jeune femme meurt elle-même peu après, mais sa mort prématurée est entourée de mystère ${ }^{10}$, mystère qui s'éclaircira au fur et à mesure de la confession du vieillard mourant. Ce sont sa fille, Kitty, et sa petite-fille, Malika, qui assistent le vieillard, mais la morte est là aussi, fantôme invisible, invisible pour tous à l'exception du «Dokter» qu'elle poursuit jusque dans ses cauchemars. Le narrateur homodiégétique unique reste le docteur, et cela même s'il transpose les paroles des deux femmes sous la forme de discours direct : tout est vu par ses yeux et rapporté par lui, la scène finale mise à part, dans laquelle une autre voix raconte la veillée imaginaire des trois femmes près du cadavre haï du vieillard. La question qui se pose est donc de savoir comment une instance narrative avec laquelle le lecteur aurait du mal à s'identifier ou de sympathiser avec elle, parvient à rendre son récit attachant tout en provoquant la désapprobation, le dégoût du même lecteur.

C'est pourquoi nous recourons au modèle théorique de Booth exposé dans son étude déjà classique, Rhétorique de la fiction (1961, 1983). Dans le chapitre VI de son ouvrage ${ }^{11}$ Booth introduit la notion d'implied author et il définit l'auteur implicite comme l'alter ego de l'auteur réel en quelque sorte, un metteur en scène, ou, comme le dit bien plus tard Anaïs Oléron, « un garant invisible [...] des normes du texte» (Oléron, 2018). Le critique américain propose également certaines classifications des narrateurs en fonction du degré et de la nature de leur présence pour ainsi dire palpable dans l'œuvre ; ainsi les narrateurs peuvent être "non représentés » ou "représentés »; ces derniers peuvent être des agents-narrateurs ou des observateurs, etc. Booth traite ensuite la question «du degré et du type de distance » (Booth, 1977 : 100) - morale, intellectuelle, physique et temporelle - qui peut exister entre le narrateur et l'auteur implicite, le narrateur et les autres personnages de l'histoire ou, enfin, entre le narrateur et le lecteur, qui mènent tous un « dialogue implicite » (Booth, 1977 : 101). De surcroît, il précise que l'auteur

10 Selon la famille, très discrète sur les causes de sa mort, elle semble être morte pratiquement en couches. Plus le récit avance, plus la vérité va se faire jour, que le corps de la jeune femme - qui s'est de toute manière laissée mourir - a été brûlé sans qu'il y ait certitude par qui.

${ }^{11}$ Ce chapitre a été traduit et publié dans un ouvrage collectif, cf. Booth, 1977. 
implicite, lui aussi, peut se trouver à une distance plus ou moins grande du lecteur ou des personnages de son œuvre. Cependant, le critique confère une importance primordiale à la distance qui peut s'établir entre l'auteur implicite et son narrateur et il propose les termes de reliable/unreliable (selon les traductions «fiable/non fiable» ou encore «digne/indigne de confiance ») pour qualifier le narrateur qu'il définit de la manière suivante :

Par manque de meilleurs termes, je dirai d'un narrateur qu'il est digne de confiance (reliable) quand il parle ou agit en accord avec les normes de l'œuvre (ce qui revient à dire : avec les normes implicites de l'auteur), et je le dirai indigne de confiance (unreliable) dans le cas contraire [...] (Booth, 1977 : 105).

Comme on peut le constater, c'est le rapport aux normes de l'œuvre et à l'auteur implicite qui est essentiel ${ }^{12}$, mais Booth tient aussi à préciser que ce rapport peut s'établir de différentes manières :

Être indigne de confiance ne signifie pas mentir, bien qu'une des principales ressources pour certains romanciers modernes ait été d'utiliser des narrateurs délibérément menteurs [...] C'est une attitude généralement liée à ce que James appelle l'inconscient ; le narrateur se méprend, ou il prétend à des qualités que l'auteur lui refuse. Ou bien, comme dans Huckleberry Finn, le narrateur proclame qu'il est tout simplement affreux, alors que l'auteur, dans son livre, loue ses vertus en silence » (Booth, $1977:$ 105).

L'auteur implicite et le narrateur ne partagent donc pas nécessairement les mêmes valeurs, bien au contraire. Aux exemples fameux cités par Booth on ajoute fréquemment des exemples contemporains, tel le roman Les Bienveillantes (2006) de Jonathan Littel dans lequel les «valeurs » d'un nazi particulièrement odieux et schizophrène, Maximilien Aue, ne peuvent être partagées ni par l'auteur implicite ni d'ailleurs par le lecteur moyen, à moins

12 Avant de définir le narrateur in/digne de confiance, Booth constate : "Quand on parle de point de vue en fiction, la distance la plus gravement négligée parmi toutes est celle située entre le narrateur capable d'erreur, ou indigne de confiance, et l'auteur implicite, ce dernier entraînant le lecteur avec lui, aussi bien que contre le narrateur » (Booth, 1977 : 105). 
qu'il n'ait des convictions nazies ${ }^{13}$. Cependant, d'après Booth, il est possible de remédier, du moins partiellement, à la non-fiabilité du narrateur, avec «le soutien et le correctif qu'apportent d'autres narrateurs » (Booth 1977 : 107). Et le critique précise qu'il y a deux manières de corriger un narrateur : « de l'intérieur de l'action » pour aider le narrateur à en tirer avantage ou "de l'extérieur, de façon à aider le lecteur à corriger ou à établir fermement ses propres vues, qui vont à l'encontre de celles du narrateur » (Booth 1977 : 107, l'italique est de l'auteur).

Certains chercheurs toutefois estiment que les thèses de Booth restent à nuancer quant à la définition même de la (non) fiabilité du narrateur, alors que d'autres considèrent que ce critique accordait trop d'importance à l'auteur implicite - d'après eux vaguement défini - au détriment des normes éthiques du lecteur, autrement dit, de la réception du texte ${ }^{14}$. Quelque peu au croisement des deux tendances, dans un texte publié en 1999, Ansgar Nünning insiste d'abord sur la différence entre la non fiabilité factuelle d'un narrateur (faisant référence à l'appellation de unreliable, non fiable, capable d'erreur) et la non fiabilité idéologique (untrustworthy, dont les valeurs, les normes morales, sont inacceptables (dans Wagner, 2016 : 156). Nünning attire aussi l'attention sur l'importance de l'attente du lecteur quand un jugement sur la (non) fiabilité du narrateur doit être porté : les contextes historiques et culturels et d'autres facteurs influencent grandement notre jugement et non pas uniquement les normes de l'œuvre ou de l'auteur implicite - même s'il y a "un garde-fou», que sont les valeurs communes à la majorité des êtres humains (Cf. Oléron, 2018). Des taxonomies plus compliquées du narrateur

13 C'est justement le point à propos duquel certains chercheurs adressent des critiques à la théorie de Booth, soutenant qu'il prend moins en compte les normes éthiques du lecteur que celles de l'œuvre, v. supra. Ansgar Nünning dit par exemple: «To put it quite bluntly: A pederast would not find anything wrong with Nabokov's Lolita; a male chauvinist fetishist who gets his kicks out of making love to dummies is unlikely to detect any distance between his norms and those of the mad monologist in Ian McEwan's 'Dead As They Come'." (Nünning, 1999 : 61 in Shen, 2011). Oléron soutient qu'il s'agit d'un personnage à la fois non fiable (parce que sa perception des événements est erronée) et antipathique (en raison des valeurs qu'il adopte et le manque d'empathie envers Lolita (Cf. Oléron, 2018).

14 En gros, à l'heure de la « narratologie post-classique » (notion introduite par David Herman), la différence s'est établie entre la tendance rhétorique et celle cognitiviste dans le traitement de la question (Cf. Shen, 2011, cf. aussi Baroni, 2018). 
(in)digne de confiance ont été élaborées par d'autres chercheurs, comme James Phelan, universitaire américain, qui distingue trois axes selon lesquels l'on peut décider de la (non) fiabilité du narrateur: l'axe narratif (reporting), l'axe interprétatif (interpreting) et l'axe axiologique (evaluating) ${ }^{15}$. Son schéma se complique ensuite de sous-catégories, difficiles à distinguer entre elles, mais il reste que la division en trois axes qu'il propose : le plan des faits, celui de l'interprétation et celui des valeurs propres au narrateur, nous permettent de nuancer encore mieux la répartition entre les narrateurs non fiables et ceux indignes de confiance, proposée par Nünning.

Une fois toutes ces nuances prises en compte, lorsqu'on analyse le narrateur du Sari vert, l'on constate qu'il tient en haute estime l'honnêteté qui est une qualité, d'après lui, souvent absente du monde qui l'entoure et que lui cultive presque à outrance : ainsi, il proclame haut et fort, et à maintes reprises, qu'il voudrait se libérer des «faux-semblants » imposés par la société tout entière, société qui n'a d'estime que pour les apparences. Sa confession commence de la manière suivante : "Je ne suis pas l'apôtre du dire poli. Je ne souscris pas à l'hypocrisie de ces belles et vides formules dont notre époque est si friande » (Devi, 2009: 9). Il s'engage donc à dire, à raconter toute la vérité.

Dans son monologue, le vieillard se met à dénoncer les faux-semblants de cette société qu'il se considère capable de juger : il pointe du doigt surtout les femmes qui ne sont qu'une illusion, tout autant que la vie conjugale, « comme un palais aux infinies promesses où l'on ne découvre une fois la porte enfoncée, que la vétusté et le vide » (Devi, 2009 : 25). De surcroît, le médecin n'est pas inconscient non plus des défauts de son propre caractère ni de sa propre perversion et en parle ouvertement. Il se sait machiste, mais choisit d'en rire avec condescendance (Devi, 2009 : 105-107) ; lorsqu'il lève la main sur sa femme pour la battre, il concède qu'il s'agit d'un « acte violent » (Devi, 2009 : 31). Il avoue également sa compétence toute relative en médecine (Devi,

${ }^{15}$ La traduction de ces termes reprise du texte d'Oléron (2018). Et voici l'explication de Shen: "Phelan points out that narrators 'perform three main roles-reporting, interpreting, and evaluating; sometimes they perform the roles simultaneously and sometimes sequentially' (2005 : 50). In light of these three roles, Phelan classifies unreliability by focusing on three axes: the axis of facts; the axis of values or ethics; and the axis of knowledge and perception, the last having received less attention from Booth than the other two axes » (Shen, 2011). 
2009 : 98). La lucidité et la franchise semblent donc être ce qui le définit le mieux.

Cependant, le lecteur détecte immédiatement aussi une duplicité continuelle chez le docteur : elle est d'abord une stratégie dans sa lutte contre la société où il cultive "l'apparence des mots, l'apparence du savoir, l'apparence de la confiance, l'apparence des épaules carrées malgré ma petite taille, l'apparence d'une personnalité et d'une aura d'importance. » (Devi, 2009 : 49). En tant que médecin, il est entouré de «vénération » (Devi, 2009 : 41) qu'il accueille avec plaisir, tout en connaissant la vérité sur son propre savoir-faire. Tout aussi graves sont ses stratégies de manipulation à l'égard de sa propre famille. Il feint des sentiments qu'il n'a pas: "J'en rajoute en soupirant et en feignant une larme » (Devi, 2009 : 23). Il dit parfois aussi des choses qu'il ne pense pas et ment sans vergogne sur des sujets graves. Un exemple particulièrement saisissant est le stratagème qu'il déploie devant Malika qui consiste à inventer une maladie nerveuse de Kitty, soutenant que celle-ci est maniaco-dépressive ; ce mensonge se double d'un chantage affectif et du conseil donné à Malika de faire hospitaliser sa mère de force, pour son bien ; par dessus le marché, voyant une lueur d'inquiétude dans les yeux de sa petite-fille, il s'en réjouit (Devi, 2009: 88-91) ${ }^{16}$. Là dessus, le Dokter est cependant entièrement franc face au lecteur auquel il livre ses pensées les plus noires et dévoile ses pires agissements. Que le narrateur du Sari vert soit extrêmement antipathique, cela ne peut faire aucun doute. Les contradictions de son caractère sont-elles suffisantes pour le déclarer non fiable ou indigne de confiance?

C'est ici qu'il faut examiner le second critère établi par Phelan, celui de l'interprétation des faits. Que le narrateur oscille entre honnêteté et fourberie et qu'il le rapporte, cela ne l'empêche donc pas de porter des jugements non fiables, erronés, et de se méprendre tout d'abord quant à sa propre personne : le docteur se croit pratiquement omniscient et infaillible et doté de nombreuses qualités. Il développe un sentiment de supériorité démesurée et se targue, alors qu'il se sait mourant et vieux, d'avoir gardé « la svelte élégance et la classe naturelle », contrairement à sa fille au "visage décati, trop pâle, aux ridules visibles» (Devi, 2009: 20) et à sa petite-fille qu'il qualifie de

16 Ajoutons qu'il se trompe et quelques instants plus tard constate que Malika est plus forte qu'il ne le croyait, un signe de sa faillibilité. 
« maquerelle [...] avec son teint noir, ses membres épais, ses gros doigts » (Devi, 2009 : 78). Sur le plan moral, le même discours prétentieux et irréaliste est déployé : "J'ai vécu une vie exemplaire, mais toutes ces femmes en ont déformé le sens, altéré la droiture. J'avais tant de choses à leur apprendre. Elles n'ont pas compris que j'étais un héros (Devi, 2009: 35). Inutile de dire à quel point le réel bouscule les certitudes du docteur : ses actions, aussi bien que les paroles et les réactions des deux femmes qu'il rapporte, contredisent une telle image de lui.

Encore plus signifiants sont les jugements erronés portés par le docteur sur les comportements et les réactions des membres de sa famille au présent : lorsqu'il croit sans difficulté parvenir à lire les pensées de sa fille, qui, d'après lui, souffre de «la logorrhée ophtalmique» (Devi, 2009: 79), elle va au contraire, surtout dans les scènes finales du roman, révéler toute la turpitude morale de son père, se rappelant les pires avanies commises par lui et les étalant au grand jour (Cf. notamment Devi, 2009: 236-240). Ou encore, ressassant le passé, le narrateur interprète les maladies psychosomatiques de Kitty comme de la comédie pure et simple : «Elle parvient à se faire son cinéma pour se donner de l'importance, se terrassant de maladies qui l'empêchaient de grandir et de se développer. » (Devi, 2009 : 45). Tout comme, d'après lui, une fois devenue adolescente, elle n'a qu'un seul objectif, celui de le manipuler (Devi, 2009 : 108). De la même manière sont commentés les agissements de la femme du docteur : lorsqu'elle s'évanouit sous les coups qu'il lui donne : «elle faisait semblant d'être complètement anéantie [...] elle a fait semblant de s'évanouir. Je l'ai laissée faire pour voir jusqu'où irait la comédie [...] elle était parfaite dans son martyre» (Devi, 2009 : 147-148). Et le docteur d'évoquer tous les travers de sa femme, dont celui d'être une très mauvaise ménagère et de ne pas repriser ses caleçons, pour conclure: «Elle devait bien rigoler en pensant à moi marchant dans mes sous-vêtements de pauvre» (Devi, 2009: 151, l'italique est partout de nous dans ce paragraphe).

Le comble est atteint cependant par l'exposé des valeurs que défend le docteur et qu'il serait difficile d'approuver, encore plus d'adopter. Même lorsqu'il nous semble entendre dans ses paroles des accents de compassion 
pour la souffrance des plus démunis ${ }^{17}$, il s'avère que cela lui sert uniquement pour mieux maltraiter son propre enfant et lui apprendre que la vie est dure :

Kitty serait une fille comme les autres. Elle n'était pas une princesse. Dans ce pays où la pauvreté tuait chaque jour des centaines d'humains, il n'y avait pas de princes. Il n'y avait que des hommes. [...] j'avais pour but la droiture » (Devi, 2009: 74).

Lorsqu'on considère le traitement que le docteur réserve à sa femme et à sa fille ${ }^{18}$, son profond mépris pour la gente féminine en général est on ne peut plus logique: les femmes sont rabaissées au rang des animaux, appelées « femelles » (Devi, 2009 : 58-59) et ne doivent aspirer à aucun droit : « Ce n'est que plus tard que les hommes sont devenus des mauviettes et que les femmes ont eu des droits » (Devi, 2009 : 27). Dans le même ordre d'idées, le docteur n'a aucune difficulté pour avouer son intolérance à l'égard des noirs: apprenant que Malika vit avec une femme et non avec un homme, ce qui est déjà inacceptable pour lui, et apprenant en plus qu'il s'agit d'une femme noire, Marie-Rose, il tient à s'exprimer sur le bien-fondé de l'esclavage, soupçonnant les noirs de posséder « une sorte de paresse si profonde qu'il fallait l'esclavage pour les faire travailler mais bon, je vais encore me faire traiter de tous les noms parce que je dis ces vérités-là. » (Devi, 2009: 61). Par les valeurs que défend le docteur, il peut être qualifié d' «indigne de confiance» (untrustworthy), sa normalité étant difficilement défendable.

Il s'agit donc d'un personnage profondément antipathique, violent et cruel. La plupart du temps il semble relativement honnête quant à la relation de ses faits et gestes, mais un élément clé a jusque ici été occulté par nous et dissimulé par lui : il s'agit des raisons et des circonstances de la mort de sa

17 L'amour inconditionnel envers sa mère (Devi, 2009 : 96-97), un certain réalisme dans la représentation de l'exploitation et de la lutte sociale à Maurice (Devi, 2009 : 100), les souvenirs de sa propre misère et souffrance (Devi, 2009 : 112) sont les rares moments où l'on identifie des restes d'humanité chez le docteur.

18 Lorsque sa femme meurt il n'a que des mots durs pour son propre enfant: « Me laissant sur les bras cette chose dont je n'avais que faire» (Devi, 2009 : 56). Il utilise par ailleurs nombre d'autres appellations dénigrantes pour la nommer. Cependant, il persiste à croire dans sa propre bonté et droiture, sans aucune empathie pour l'enfant : "J'étais un père plutôt attentif. Mais j'attendais d'elle un minimum d'intelligence, un éclair d'esprit qui m'auraient rendu fier d'elle » (Devi, 2009 : 43-44). 
femme. C'est à ce propos que le "Dokter Dieu», sous la pression des deux femmes, tente d'entreprendre son mensonge suprême, celui de faire croire à la culpabilité de sa propre fille, Kitty. Par le passé, il l'avait persuadée de la sorcellerie de sa propre mère et la pretend maintenant coupable d'avoir tué celle-là avec des allumettes laissées par le docteur au chevet de la jeune femme. Le correctif va ici fonctionner avec succès, Kitty parvient à se rappeler ce qu'elle avait vécu et le crie à son père avant de l'abandonner pour toujours ${ }^{19}$, se vengeant ainsi sur le monstre pour les souffrances infligées à sa mère, elle-même et, indirectement, à sa fille : « elle est partie avant que tu ne jettes ton allumette encore enflammée sur le pan de son sari » (Devi, 2009: 239-240). Les faits racontés par le docteur sont donc «contredits par un témoignage » (Booth 1977: 107), son discours est miné, il est déjoué et durement sanctionné.

Nous avons donc démontré que la vérité que nous livre le vieillard sur ses faits et gestes est toute relative : il est tout d'abord non fiable parce qu'il ment ou déforme les faits qu'il raconte (reporting), nous venons d'en voir la preuve ultime, le mensonge sur la façon dont sa femme est morte. De plus, il ne parvient pas à bien percevoir le point de vue et les motivations des autres personnages, à les «lire », ou s'il le fait, il se convainc de ce qui l'arrange le mieux afin de trouver un moyen de se dédouaner, se blanchir et de se présenter sous un jour avantageux en accord avec ses idées hautement présomptueuses sur son propre caractère qui s'opposent manifestement à la réalité (interpreting). Enfin, le docteur est indigne de confiance sur le plan éthique (evaluating), parce que ses raisonnements et ses actions s'opposent à la morale la plus élémentaire. Que nous adoptions la classification la plus « simple » de Booth, ou celles, plus développées de Nünning, puis de Phelan, le vieillard reste désespérément indigne de confiance. Quant à l'auteur

\footnotetext{
19 L'émancipation des deux femmes, leur jugement sévère, leur rejet de ce père et grand-père monstre ne peuvent ici être analysés en détail. Pour appuyer nos affirmations sur le correctif qu'elles représentent, nous citons seulement deux des répliques, celle de Kitty : "C'est ça un père ? » et, un peu plus loin, elle demande, avec dégoût : "C'est donc ça, un homme ? (Devi, 2009: 80, 82) ; Malika pour sa part, à propos de la douleur physique que son grand-père ressent, dit: "Tu as mal à toimême, c'est tout», et quelques instants après, lui demande : "mais comprends-tu pourquoi nous te haïssons? » (Devi, 2009 : 87).
} 
implicite ${ }^{20}$, eu égard à tous les signaux de cette entreprise de "minage » que nous venons de présenter, il semble bien enfoncer son narrateur dans la « sublime nullité de l'obscur » (Devi, 2009 : 62).

Tamara Valčić Bulić

ON EFFECTS OF THE (UN)RELIABLE NARRATOR OF MAURITIAN «THE KNOT OF VIPERS »

\section{Summary}

The Green Sari (Le Sari vert, 2009) by Ananda Devi, a novel about domestic hatred and abuse, revolves around a family of Indian origin living in Mauritius. The novel is written as an internal monologue of an old man (Dokter Dieu) who, in his final hours, is being cared for by his daughter and granddaughter. The main story is, in fact, a string of memories about how his young wife mysteriously lost her life, while his daughter and granddaughter try to uncover the truth about that death. It is especially interesting that the only narrative voice in the novel is that of the old man, so the only insight the reader gets into the other two characters is through his interpretation of the words they spoke. Therefore the focus of this study is the question - how and by what means does the implied author manage to simultaneously make the narrator unreliable as

20 Il va sans dire que nous avons complètement omis ici d'évoquer les intentions d'Ananda Devi, étant donné que l'auteur implicite est une figure qui construit le texte, mais n'est pas pour autant le double de l'auteur réel. Cependant, Devi s'exprime sur son choix du narrateur masculin et les effets de ce choix d'un personnage à la fois monstrueux et captivant, aussi bien sur elle-même que sur les lecteurs, sans pour autant le définir comme non fiable ou indigne de confiance: «À partir du moment où j'ai pensé à la raconter avec la voix de l'homme, l'écriture a été très facile. Paradoxalement - parce que c'est une histoire très dure - je me suis trouvée presque possédée par cet homme. Je l'ai écrite d'une façon très rapide, très fluide. Je suis rentrée dans sa logique, dans son esprit. [...]. Le lecteur est en rapport avec cet homme, avec le narrateur qui n'est jamais indifférent ; on se sent pris par lui. Soit on est captivé ou captif, soit on a envie de jeter le livre et en même temps on veut savoir ce qui se passe... ». http://ile-en-ile.org/ananda-devi-5-questions-pour-ile-en-ile/ 
well as repulsive to the reader? The theoretical basis for this comprises terms implied author and (un)reliable narrator, first used by the American critic W. Booth in his, now classic study, Rhetoric of Fiction (1961). Later theoreticians introduce further nuances into Booth's categorization e.g. A. Nünning (1999) who suggests differentiating between the unreliable narrator (who has his/her facts wrong) and the untrustworthy narrator (due to unacceptability of his/her moral norms). An American university professor, J. Phelan, further adds criteria for categorizing (un)reliable narrators and establishes three axes upon which we can determine narrator's (un)reliability: reporting, interpreting and evaluating. Using these theoretical terms, we have analyzed the narrator in the novel The Green Sari. Though with a few contradictions in his account, it can be said that the narrator, to a great extent, openly discussed even his most horrid acts (family violence), except that concerning his wife's death. Fortunately, this is corrected by the memory of his daughter, who accuses the old man. When it comes to interpretation, though striving to be omniscient, the narrator often makes mistakes both when judging his own personality and understanding the psychological traits and motivations of other characters. Finally, the values that the narrator promotes (e.g. misogyny, racism) are completely unacceptable. Therefore, the implied author achieves his goal of showing a monster through different cues of the narrator's unreliability.

Key words: Ananda Devi, Le Sari vert, implied author, (un)reliable narrator, (un)trustworthy, reporting, interpreting, evaluating

\section{RÉFÉRENCES BIBLIOGRAPHIQUES}

Baroni, R. (2018). Narratologie postclassique / Postclassical Narratology. (2018, 10 septembre). In : Glossaire du RéNaF. Consulté le 25 juillet 2021, sur https://wp.unil.ch/narratologie/2018/09/narratologiepostclassique-postclassical-narratology/

Booth, W. C. (1977). Distance et point de vue. In: Genette G.-Todorov T. (dir.) (1977). Poétique du récit / R. Barthes, W. Kayser, W. C. Booth, Ph. Hamon. Paris : Seuil. 85-113.

Corio, A. (2005). Entretien avec Ananda Devi. Francofonia, No. 48, La littérature mauricienne de langue française (Printemps / Primavera 2005), 145-167.

Devi, A. (2009). Le Sari vert. Paris : Gallimard.

Garcia, M. (2007). Entretien avec Ananda Devi. (2007, 27 août). In : La Tortue Verte, Revue en ligne des Littératures Francophones. Disponible sur http://www.latortueverte.com/Entretien\%20avec\%20Ananda\%20De vi.pdf 
Oléron, A. (2018). Narrateur non fiable et effets de valeurs : pour ou contre une nette démarcation entre narrateurs non fiables et narrateurs antipathiques. Consulté le 20 juillet 2021, disponible sur: https://hal.ird.fr/CRIMEL/hal-01839926v1

Panisse, M. (2015). L'expression de la violence et de la souffrance dans trois romans d'Ananda Devi. In : S. Á. Eíriksdóttir (red.) (2015). Actes du XIXe Congrès des Romanistes Scandinaves (s.-). Consulté le 5 février 2021, disponible sur : https://rafhladan.is/bitstream/handle/10802/11870/ MIAPANISSE.pdf?sequence $=18$

Shen, D. Unreliability. (2011, 27 June). In: Hühn, Peter et al. (eds.) (2011). the living handbook of narratology. Hamburg : Hamburg University. Consulté le 10 juillet 2021. Disponible sur: https://www.lhn.unihamburg.de/node/66.html

Spear, T.C. (1er juin 2013). Ananda Devi, 5 Questions pour Île en île. [vidéo]. Disponible sur: http://ile-en-ile.org/ananda-devi-5-questions-pour-ileen-ile/

Wagner, F. (2016). Quand le narrateur boit(e)... (Réflexions sur le narrateur non fiable et/ou indigne de confiance). Arborescences, 6, 148-175. https://doi.org/10.7202/1037508ar

Wiśniewska, K. (2011). Les personnages marginalisés dans les romans d'Ananda Devi. Romanica Silesiana, 6 (1). 303-320. Consulté le 13 mars 2021, disponible sur: https://journals.us.edu.pl/index.php/RS/ article/view/5806 
